This item was submitted to Loughborough's Research Repository by the author.

Items in Figshare are protected by copyright, with all rights reserved, unless otherwise indicated.

\title{
A modified boundary condition of velocity for continuity equation with non- uniform density distribution at outlet boundary plane
}

PLEASE CITE THE PUBLISHED VERSION

https://doi.org/10.1252/jcej.17we178

\section{PUBLISHER}

(C) The Society of Chemical Engineers, Japan

\section{VERSION}

VoR (Version of Record)

\section{PUBLISHER STATEMENT}

This work is made available according to the conditions of the Creative Commons Attribution-NonCommercialNoDerivatives 4.0 International (CC BY-NC-ND 4.0) licence. Full details of this licence are available at: https://creativecommons.org/licenses/by-nc-nd/4.0/

\section{LICENCE}

CC BY-NC-ND 4.0

\section{REPOSITORY RECORD}

Yokoi, S., Yoshiya Matsushita, Y. Saito, Yohsuke Matsushita, H. Aoki, and Weeratunge Malalasekera. 2019. "A Modified Boundary Condition of Velocity for Continuity Equation with Non-uniform Density Distribution at Outlet Boundary Plane". figshare. https://hdl.handle.net/2134/34623. 


\title{
A Modified Boundary Condition of Velocity for Continuity Equation with Non-uniform Density Distribution at Outlet Boundary Plane
}

\author{
Satoki Yokor ${ }^{1,2}$, Yoshiya Matsukawa ${ }^{1}$, \\ Yasuhiro SAIto ${ }^{1}$, Yohsuke Matsushita ${ }^{1}$, Hideyuki Aoki ${ }^{1}$ and \\ Weeratunge MALALASEKERA ${ }^{3}$ \\ ${ }^{1}$ Department of Chemical Engineering, Graduate School of Engineering, Tohoku University, \\ 6-6-07 Aoba, Aramaki, Aoba-ku, Sendai, Miyagi 980-8579, Japan \\ ${ }^{2}$ Research Fellow of Japan Society for the Promotion of Science, \\ ${ }^{3}$ Wolfson School of Mechanical, Electrical and Manufacturing Engineering, Loughborough University, \\ Loughborough, Leicester LE11 3TU, United Kingdom
}

Keywords: Outflow Boundary Condition, Finite Volume Method, Unsteady State, Collocated Grid

Boundary conditions in computational fluid dynamics significantly affect the prediction of flow field. However, the outlet boundary conditions for the continuity equation have been rarely investigated. In addition, the velocities at the outlet boundary might not be accurately predicted with the conventional outlet boundary conditions when a flow that has non-uniform density distribution on the outlet boundary is simulated. In the present study, we modified a boundary condition for the continuity equation in consideration of the non-uniform density distribution on the outlet boundary plane, comparing the numerical results of combustion between the conventional and modified boundary conditions. As a result, the proposed boundary condition can resist the generation of an unrealistic temperature field better than the conventional methods.

\section{Introduction}

Boundary conditions in computational fluid dynamics significantly affect the prediction of flow field, and they have been widely studied. As for the outlet boundary, Orlanski (1976) applied the convection equation to the outlet boundary condition instead of the zero-gradient velocity condition to avoid the undesired distortion of the flow field near the outlet boundary. Moreover, the convection velocity in the convection equation were modified to be the second-order accuracy in time (Han et al., 1983) and to improve the robustness and accuracy in large eddy simulation (Dai et al., 1994). As mentioned above, there are many studies on the outlet boundary conditions for the momentum conservation equations. However, the outlet boundary conditions for the continuity equation have been rarely investigated.

Matsushita (2011) proposed an outlet boundary condition for the continuity equation as an equation for pressure correction based on a SIMPLE algorithm in low Mach number flow simulation. He introduced an imbalance between the outlet mass flow rate calculated from the summation of the discretized continuity equation for all cells and that calculated from the discretized momentum conservation equations, corrected velocities at the outlet boundary using

Received on May 30, 2017; accepted on February 27, 2018

DOI: $10.1252 /$ jcej.17we178

Correspondence concerning this article should be addressed to S. Yokoi (E-mail address: $000 @ 000)$. the imbalance of mass flow rate, and used the mass flow rate given by the corrected velocities as the outlet boundary condition for the continuity equation. Then, this boundary condition can be regarded as a velocity correction equation at the outlet boundary. Using this boundary condition, he showed that stable and robust, and accurate numerical simulation for variable-density fluid flows can be performed. In addition, Matsushita et al. (2014) reported that numerical simulations using a collocated grid instead of a staggered grid and this boundary condition showed also good convergence performance and conservativeness. At this boundary condition, the density distribution on the outlet boundary plane was assumed to be uniform, and thus the velocity for compensating the imbalance of the mass flow rate was equally added to each velocity at the outlet boundary. Ideally, the outflow boundaries should be placed sufficiently downstream not to affect the flow field in an analytical domain. In this instance, the above-mentioned conventional boundary condition worked well. However, in numerical simulations like an open flame in a limited analytical domain, non-uniform density distributions are usually generated on the outlet boundary planes. Therefore, the velocities at the outlet boundary might not be accurately predicted with the conventional boundary condition due to the assumption that the density distribution on the outlet boundary plane was uniform, and then the momentum near the outlet boundary should unrealistically increase or decrease.

In the present study, we modified a boundary condition for the continuity equation in consideration of the non- 
uniform density distribution on the outlet boundary plane. At this proposed boundary condition, a mass flux instead of the velocity at the outlet boundary was corrected with the imbalance of mass flow rate, and the velocities at the outlet boundary were determined with the corrected mass flux. Comparing the numerical results with the conventional and modified boundary conditions, the validity of the modified boundary condition was investigated.

\section{Methods}

\subsection{Boundary condition for the equation for pressure correction}

In low Mach number flow, the mass flow rate at the outlet was determined from the summation of the discretized continuity equation for all of the cells in the analytical domain (i.e. the mass flow rates at the inlet and the accumulation of the expansion or compression) as Eq. (1) below.

$$
F_{\mathrm{O}}-F_{\mathrm{I}}+\sum \frac{\rho_{\mathrm{P}}^{n+1}-\rho_{\mathrm{P}}^{n}}{\Delta t} \Delta V_{\mathrm{P}}=0, \text { where } F=\sum \rho_{\mathrm{P}, i}^{n+1} u_{i} A_{i}
$$

Because velocities calculated from the discretized momentum equations might not necessarily satisfy the discretized continuity equation, the outlet mass flow rates calculated with the discretized momentum equation was different from that with the summation of the discretized continuity equation for all of the cells in the analytical domain. The imbalance was given by Eq. (2).

$$
F_{\mathrm{O}}^{*}-F_{\mathrm{I}}+\sum \frac{\rho_{\mathrm{P}}^{n+1}-\rho_{\mathrm{P}}^{n}}{\Delta t} \Delta V_{\mathrm{P}}=R
$$

Herein, $F_{O}^{*}$ is the outlet mass flow rate calculated from the discretized momentum equations, and $R$ is the imbalance of the mass flow rate. Matsushita et al. (2014) subtracted Eq. (2) from Eq. (1) to eliminate $R$, converted the imbalance of the mass flow rate to the compensating velocity by dividing the imbalance by the density averaged over the cross-sectional region and the area of outlet, and added this velocity to the outlet velocities as the following Eq. (3) when pressure correction equations are solved to improve the conservativeness.

$$
u_{i, \mathrm{O}}^{n+1} \cong u_{i, \mathrm{O}}^{*}-\frac{F_{\mathrm{O}}^{*}-F_{\mathrm{I}}+\sum \frac{\rho_{\mathrm{P}}^{n+1}-\rho_{\mathrm{P}}^{n}}{\Delta t} \Delta V_{\mathrm{P}}}{\left.\sum \rho_{\mathrm{P}, i}^{n+1} A_{i}\right|_{\mathrm{O}}}
$$

It can be considered that this outlet boundary condition corrects the outlet velocities to satisfy the discretized continuity equation and a Neumann boundary condition for the velocities. This attribution is appropriate when the outflow boundary is placed downstream so that the density distribution is uniform. However, when the density distribution on the outlet boundary plane is non-uniform, an unrealistic flow field can be predicted with the outlet boundary condition of Matsushita et al. because the outlet fluid can be accelerated or decelerated due to the addition of equal velocity calculated by the imbalance of mass flow rate regardless of the non-uniform density distribution, as shown in Figure 1.

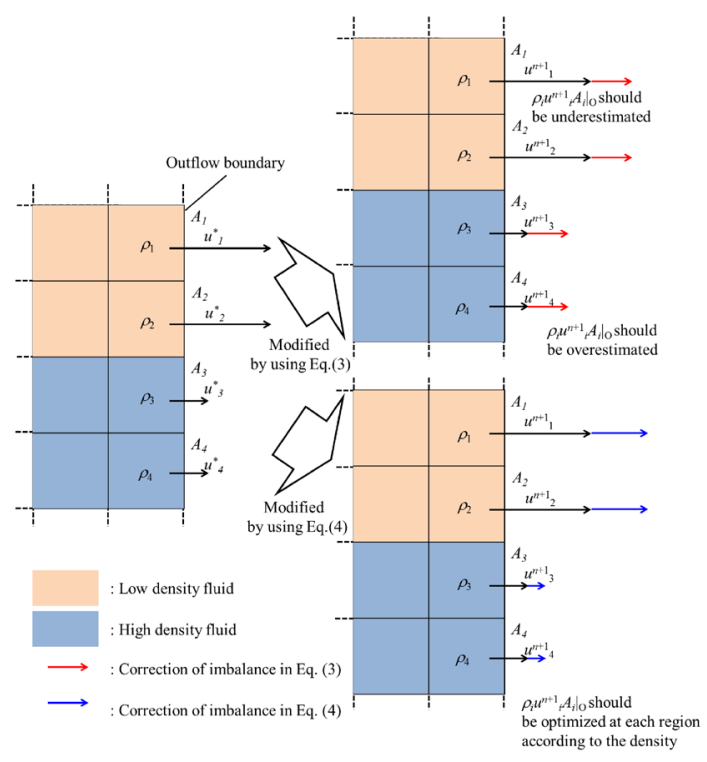

Fig. 1 Conceptual diagram of the outlet boundary conditions

Hence, in the present study, a modified boundary condition was proposed to take the non-uniform density distribution into account, and can be written as Eq. (4).

$$
u_{i, \mathrm{O}}^{n+1} \cong u_{i, \mathrm{O}}^{*}-\frac{F_{\mathrm{O}}^{*}-F_{\mathrm{I}}+\sum \frac{\rho_{\mathrm{P}}^{n+1}-\rho_{\mathrm{P}}^{n}}{\Delta t} \Delta V_{\mathrm{P}}}{\left.\rho_{\mathrm{P}, i}^{n+1} \sum A_{i}\right|_{\mathrm{O}}}
$$

In this proposed boundary condition, the mass fluxes for compensating the imbalance of mass flow rate were added to the outlet mass fluxes, and the velocities at the outlet were computed with the mass fluxes to be corrected. The point of the proposed condition is that the velocities calculated from the mass fluxes corrected by $R$ are used instead of the velocities directly corrected by $R$.

This outlet boundary condition corrects the outlet velocities to satisfy the discretized continuity equation and a Neumann boundary condition for the mass fluxes instead of the velocities.

\subsection{Flamelet/progress variable (FPV) model}

In the present study, the FPV model (Pierce and Moin, 2004) was employed in combustion simulation. Before the combustion simulation, a database for the FPV model was prepared. The database was generated by FlameMaster (Pitsch, 1998) with GRI-Mech 3.0 (Smith et al., 2000), which is a detailed chemical reaction mechanism consisting of 325 reactions and 53 species. As the boundary conditions of chemical species, the composition of fuel was assumed as $\mathrm{CH}_{4} / \mathrm{N}_{2}$ with a volume ratio of $23 / 77$, and that of oxidizer was $\mathrm{O}_{2} / \mathrm{N}_{2}$ with a volume ratio of $23 / 77$. The temperatures at both sides were $300 \mathrm{~K}$. The Lewis number was assumed to be unity. Radiation heat transfer was neglected. The calculations were carried out for the cases with various scalar dissipation rates, and the results were remapped by the mixture fraction and progress variable (PV) to produce the database 
$(143 \times 117)$. The progress variable was a linear combination of $\mathrm{CO}, \mathrm{CO}_{2}, \mathrm{H}_{2}$, and $\mathrm{H}_{2} \mathrm{O}$ mass fraction.

\section{Numerical Simulation of Combustion}

A laminar counter-flow diffusion flame by Sung et al. (1995) was simulated for the representative case of the nonuniform density distribution on the outlet boundary plane. Figure 2 shows the analytical object. The oxidizer and fuel flowed from the top and bottom of the computational region with a velocity of $0.255 \mathrm{~m} / \mathrm{s}$, respectively. As the analytical object was assumed to be two-dimensional, the slip condition was applied for the boundary conditions in the depth direction. In this work, Eq. (4), Eq. (3), and the ordinary condition for SMAC (Amsden and Harlow, 1970) were used as the outlet boundary conditions to carry out the case study as shown in Table 1. In this study, to investigate the effect of the position of the outlet boundary on the flow field, the distance between the outlets was varied at 0.005, 0.015, and $0.025 \mathrm{~m}$. An unstructured grid with collocated arrangement was employed and the computational domains were divided into 1000, 3000, and 5000 hexahedrons, respectively.

Based on the finite volume method, the continuity, momentum, mixture fraction, and progress variable (PV) conservation equations were discretized. The second-order central differencing scheme was used for the discretization of the diffusive terms of all the equations and the convective term of the momentum, and the total variation diminishing scheme (limiter function of Min-Mod) was used

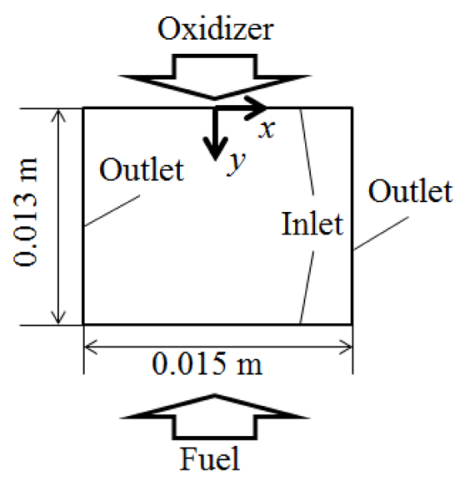

Fig. 2 Analytical object for a counter flow

Table 1 Computation cases for the combustion simulation

\begin{tabular}{lcl}
\hline Name & Distance between outlets $[\mathrm{m}]$ & \multicolumn{1}{c}{ Boundary condition } \\
\hline Case a05 & 0.005 & Proposed condition (Eq. (4)) \\
Case a15 & 0.015 & \\
Case a25 & 0.025 & \\
Case b05 & 0.005 & Conventional condition \\
Case b15 & 0.015 & (Eq. (3)) \\
Case b25 & 0.025 & \\
Case c05 & 0.005 & Original SMAC \\
Case c15 & 0.015 & (Amsden and Harlow, 1970) \\
Case c25 & 0.025 & \\
\hline
\end{tabular}

for the discretization of the convective terms of the other scalars. The second-order Adams-Bashforth method was used to advance time for the momentum equation, and the pressure-velocity coupling scheme was SMAC. The implicit Euler method was used to advance time for the other scalar equations. The simulation was conducted using the constant time step $\left(10^{-5} \mathrm{~s}\right)$ from $0 \mathrm{~s}$ (initial condition) to $0.5 \mathrm{~s}$. The density, viscosity, and temperature were determined from the database by using the mixture fraction and PV, which were the solutions of those conservation equations, according to the FPV model. The diffusion coefficient was given by the relationship between the Prandtl number and the viscosity. The Prandtl number was constant at 0.7.

\section{Results and Discussion}

\subsection{Temperature distribution}

Figure 3 presents the temperature fields computed using each outlet boundary condition at the elapsed time of $0.5 \mathrm{~s}$, at which steady-state solutions were obtained. It is demonstrated that the temperature distribution in the case b05 is stretched in the $y$-direction near the outlet boundaries. This can be explained in that the high density fluid was entrained to the outlet because the momentums in the outlet direction were estimated unrealistically higher depending on the region due to the conventional boundary condition, which assumed the uniform density distribution and added the velocity for compensating for the imbalance of mass flow rate to the velocities at the outlet boundary equally. In the case $\mathrm{c} 05$, which used the ordinary boundary condition for SMAC, the temperature field is also stretched in the $y$ direction near the outlet boundary. This is because the same problem as the case b05 would occur due to the boundary condition, whose pressure correction variables at the outlet boundary are zero as a Dirichlet condition.

To dissect the effect of the boundary condition on the reaction field, the calculated temperatures on the $y$-axis are shown in Figure 4 with experimental data (Sung et al., 1995). Note that the results in the case a 25 are representative of those in the cases a15, b25, c15, and c25 because they were nearly indistinguishable. When the distance between (a)

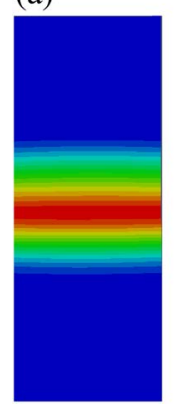

(b)

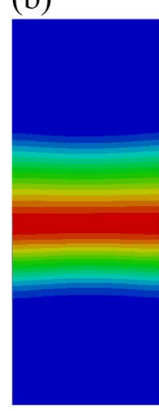

(c)

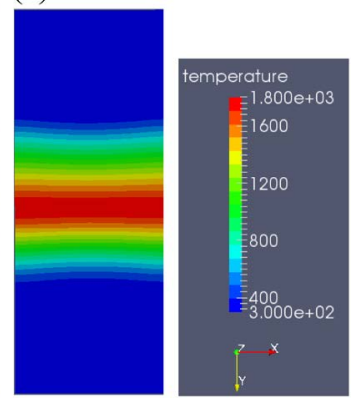

Fig. 3 Instantaneous temperature field at elapsed time $0.5 \mathrm{~s}$ using each boundary condition: (a) case a05, (b) case b05, and (c) case $\mathrm{c} 05$ 


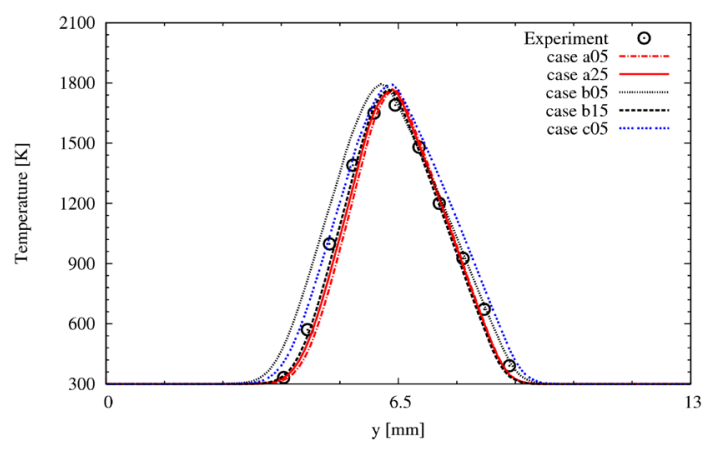

Fig. 4 Temperature distributions along $y$-axis at each distance between the outlets using each boundary condition

outlets was longer than $0.015 \mathrm{~m}$, the results mostly corresponded to each other. This indicates that when the distance between outlets is longer than $0.015 \mathrm{~m}$, the outlet boundary condition did not affect the temperature distribution along the $y$-axis. Hence, the results of the case a25 were regarded as the reasonable data, and the results in the case where the distance between outlets was $0.005 \mathrm{~m}$ were compared with those in the case a25. In Figure 4, the temperature distributions in the cases b05 and c05 were wider than that of the case a25, and the boundary conditions can affect the flow field. The temperature distribution in the case a05 was slightly narrower than that in the case a 25 due to the boundary condition. However, the difference between the cases a05 and a 25 was smaller than that between the cases b05 or c05 and a25. This reveals that Eq. (4) is suitable for the outlet boundary condition to simulate the case of the non-uniform density distribution on the outlet boundary plane.

\subsection{Velocity distribution at outlet boundary}

As the flow field should be independent of the outlet boundary, the velocity distributions at the outlet were compared with those at the corresponding location in larger analytical domain. Figure 5 shows the velocity distributions along the $y$-axis at $x=0.0025$ at elapsed times of $0.01,0.02$, 0.03 , and $0.5 \mathrm{~s}$ in each case. It is clearly observed that the results of the case a05 are in better agreement with those of the cases b15 and a25 than those of the case b05 and c05 at any time.

\subsection{CPU time}

Table 2 lists the normalized CPU times and the number of iterations to satisfy the discretized continuity equation from 0 to $0.5 \mathrm{~s}$. The CPU times in the cases $\mathrm{a} 05$ and b05 were shorter than that in the case c05. This is consistent with the tendency shown by the previous study (Matsushita et al., 2014). The CPU time in the case a05 was about $10 \%$ longer than that in the case b05. This is because of a decrease in the convergence performance according to the number of the iterations to satisfy the discretized continuity equation. The reason for the decrease should be explained by the large gradient of velocities in the $y$-direction or the physical property because the unrealistic outflow was reduced. Thus,
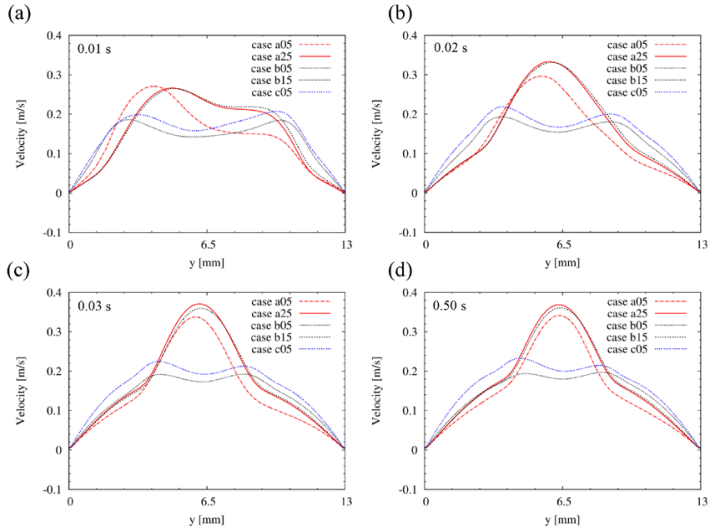

Fig. 5 Velocity distributions along $y$-axis at $x=0.0025$ at elapsed times of (a) 0.01, (b) 0.02, (c) 0.03, and (d) $0.5 \mathrm{~s}$ using each boundary condition

Table 2 Normalized CPU time at each case

\begin{tabular}{lcc}
\hline Name & Normalized CPU time & Number of iteration \\
\hline Case a05 & 0.551 & 61985 \\
Case b05 & 0.506 & 61220 \\
Case c05 & 1.000 & 301982 \\
\hline
\end{tabular}

the improvement in the calculation time with the proposed boundary condition appears in the comparison with the boundary condition of SMAC as well as that with the conventional boundary condition.

\section{Conclusion}

In the present study, we modified the boundary condition of the continuity equation as the equation of pressure correction for the non-uniform density distribution on the outlet boundary plane. The combustion simulation was carried out with the proposed and conventional boundary conditions, and the results of each condition were compared. Consequently, the modified boundary condition can resist the generation of the unrealistic temperature field better than the conventional methods. This indicates that the modified boundary condition is suitable to simulate the case of the non-uniform density distribution on the outlet boundary plane compared with the conventional boundary condition and boundary condition of SMAC.

\section{Acknowledgement}

This work was supported by JSPS KAKENHI grant number 15J04680, and part of this work was carried out under the Leading Young Researcher Overseas Visit Program of Tohoku University.

\section{Nomenclature}

$A=$ cross-sectional area $\left[\mathrm{m}^{2}\right]$

$F \quad=$ mass flow rate $\quad[\mathrm{kg} / \mathrm{s}]$

$R \quad=$ imbalance of mass flow rate between inflow and outflow boundaries 
$t=$ time

$u \quad=$ fluid velocity

$V \quad=$ cell volume

$\rho \quad=\quad$ fluid density

〈Subscripts〉

$\mathrm{I}=$ inflow boundary face

$i=$ number of face

$\mathrm{O}=$ outflow boundary face

$\mathrm{P} \quad=$ present cell on $\mathrm{i}$ th face

〈Superscripts〉

$\mathrm{n}=$ time level

* $\quad=$ intermediate

\section{Literature Cited}

Amsden, A. A. and F. H. Harlow; "A Simplified MAC Technique for Incompressible Fluid Flow Calculations," J. Comput. Phys., 6, 322-325 (1970)

Dai, Y., T. Kobayashi and N. Taniguchi; "Large Eddy Simulation of Plane Turbulent Jet Flow Using a New Outflow Velocity Boundary Condition," JSME Int J., Ser. B, 37, 242-253 (1994)

Han, T. Y., J. C. S. Meng and G. E. Innis; "An Open Boundary Condition for Incompressible Stratified Flows," J. Comput. Phys., 49, 276-297 $[\mathrm{m} / \mathrm{s}]$

$\left[\mathrm{m}^{3}\right]$

$\left[\mathrm{kg} / \mathrm{m}^{3}\right]$
(1983)

Matsushita, Y.; "Outflow Boundary Condition in the Finite-volume Method for Unsteady-state Fluid Flow Computation with Variable Density," Computational Thermal Sciences: An International Journal, 3, 531-537 (2011)

Matsushita, Y., S. Nozawa, T. Katayama, T. Soma, Y. Saito and H. Aoki; "Outflow Boundary Condition for the Unsteady-state Fluid Flow Computation with Variable Density on a Collocated Grid," Computational Thermal Sciences: An International Journal, 6, 425-437 (2014)

Orlanski, I.; "A Simple Boundary Condition for Unbounded Hyperbolic Flows," J. Comput. Phys., 21, 251-269 (1976)

Pierce, C. D. and P. Moin; "Progress-variable Approach for Largeeddy Simulation of Non-premixed Turbulent Combustion," J. Fluid Mech., 504, 73-97 (2004)

Pitsch, H.; "A c++ Computer Program for 0-D Combustion and 1-D Laminar Flame Calculations," RWTH Aachen University, Aachen, Germany (1998)

Smith, G. P., D. M. Golden, M. Frenklach, N. W. Moriarty, B. Eiteneer, M. Goldenberg, C. T. Bowman, R. K. Hanson and S. Song; J. William C. Gardiner, V. V. Lissianski and Z. Qin; "GRImech 3.0 Reaction Mechanism,” Berkeley. Available from: ‘http://www. me.berkeley.edu/gri_mech/> (2000)

Sung, C. J., J. B. Liu and C. K. Law; "Structural Response of Counterflow Diffusion Flames to Strain Rate Variations," Combust. Flame, 102, 481-492 (1995) 